\title{
Role of capecitabine in treating metastatic colorectal cancer in Chinese patients
}

This article was published in the following Dove Press journal:

OncoTargets and Therapy

2 April 2014

Number of times this article has been viewed

\author{
Feng Wang* \\ Feng-Hua Wang* \\ Long Bai \\ Rui-Hua Xu \\ Department of Medical \\ Oncology, Sun Yat-sen University \\ Cancer Center, Guangzhou, \\ People's Republic of China \\ *These authors contributed equally \\ to this work
}

Correspondence: Rui-Hua Xu Department of Medical Oncology, State Key Laboratory of Oncology in South China, Sun Yat-sen University Cancer Center, 65I Dongfeng East Road, Guangzhou 510060, People's Republic of China

Tel +86 2087343228

Fax +86 2087343468

Email xurh@mail.sysu.edu.cn

\begin{abstract}
The China Food and Drug Administration approved the use of capecitabine in patients with metastatic colorectal cancer (mCRC) in 2004. This paper reviews the available information of capecitabine in Chinese patients with $\mathrm{mCRC}$, focusing on its effectiveness and safety against mCRC. Identification of all eligible studies was made by searching the PubMed and Wanfang database from 2000 to 2013. Published data examining various aspects of clinical response and tolerability with capecitabine alone or in combination with other chemotherapeutic or biological agents for first- and second-line mCRC were examined. Capecitabine and its combination displayed high efficacy in Chinese patients with mCRC. Toxicities are generally manageable, and elderly patients can tolerate capecitabine well.
\end{abstract}

Keywords: capecitabine, metastatic colorectal cancer, Chinese

\section{Colorectal cancer in the People's Republic of China}

Colorectal cancer (CRC) is the third most common cancer in women and the fourth most common cancer in men worldwide. ${ }^{1,2}$ Previous studies have shown rapid increases in CRC incidence rates in economically transitioning countries, including most parts of Asia, Eastern European countries, and some countries in South America. ${ }^{3}$ Due to a lack of an effective national surveillance system, there has been little information available on the relationship between $\mathrm{CRC}$ and geographical environment in the People's Republic of China (PRC). However, the incidence of CRC in the PRC has increased in both urban and rural areas recently, particularly in more developed areas, such as Guangdong and Shanghai. ${ }^{4-6}$ According to a report by the Chinese Ministry of Health, the incidence of CRC has increased from the sixth to the third most common cancer, while mortality ranked fifth in all cancer types. ${ }^{7}$ The pattern of CRC has changed significantly in the last two decades, with a decrease of rectal cancer and a significant increase of proximal colon (including transverse and ascending colon) cancer. ${ }^{7}$ Because early diagnosis of CRC has shown little progress in the PRC, a lot of patients are still diagnosed at a late stage.

\section{Management of metastatic colorectal cancer}

5 -fluorouracil (5-FU) has been used to treat $\mathrm{CRC}$ for decades, and remains the backbone of CRC chemotherapy. Median survival of metastatic CRC (mCRC) was 12 months with 5-FU as the sole active agent. ${ }^{8}$ Prolonged infusion of 5-FU in combination with leucovorin regimens such as de Gramont et al and the Arbeitsgemeinschaft für Internistische Onkologie (AIO) study group achieved, improved efficacy and safety profiles compared with the bolus 5-FU/leucovorin (LV) ${ }^{9,10}$ Since 2000, with the approval of irinotecan and 
oxaliplatin, combination regimens of infusional 5-FU/LV plus oxaliplatin (FOLFOX) and infusional 5-FU/LV plus irinotecan (FOLFIRI) led to median survivals in the 15- to 20-month range. ${ }^{8}$ Although infusional 5-FU has increased therapeutic effect, long-term central venous access causes considerable inconvenience and a high rate of complications, such as thrombosis and infection. ${ }^{11}$ Under these circumstances, oral analogs of 5-FU, including tegafur-uracil, capecitabine, and S-1 have been created. These orally administered 5-FU analogs enable physicians treating $\mathrm{CRC}$ to mimic the effect of infusional 5-FU, but in a convenient outpatient setting, without the complications and costs associated with infusion pumps and parenteral therapies. In countries like the PRC, where medical resources are limited and imbalanced between urban and rural areas, oral administration of chemotherapy drugs would potentially reduce travel costs and use of health care resources.

Oral capecitabine ( $N^{4}$-pentyloxycarbonyl-5'-deoxy-5fluorocytidine, Xeloda ${ }^{\circledR}$; Roche, Basel, Switzerland) demonstrated a statistically significant superior response rate $(26 \%$ versus [vs] 17\%), equivalent time to progression (TTP) and overall survival (OS), and an improved safety profile and improved convenience compared with intravenous bolus 5-FU/LV (Mayo Clinic regimen) as first-line treatment for mCRC. ${ }^{12}$ The doublet combination of capecitabine plus oxaliplatin (XELOX) has been shown to be noninferior to FOLFOX as first-line and second-line treatment for mCRC in terms of response rate, progression-free survival (PFS), and OS in multiple studies. ${ }^{13-19}$ On the other hand, the combination of capecitabine with irinotecan (XELIRI) was inferior to FOLFIRI in terms of PFS and OS. ${ }^{20,21}$ In addition, adverse effects, including diarrhea, vomiting, and dehydration occurred more frequently in the XELIRI group than in the FOLFIRI group. ${ }^{20}$

With the advent of three humanized monoclonal antibodies that target vascular endothelial growth factor (VEGF; bevacizumab) and the epidermal growth-factor receptor (cetuximab and panitumumab), the treatment algorithms and survival for mCRC patients have changed dramatically. Another two molecularly targeted agents - intravenously administered aflibercept, ${ }^{22}$ a recombinant fusion protein consisting of human VEGF extracellular domain and the Fc portion of human immunoglobulin $\mathrm{G}_{1}$, and regorafenib, ${ }^{23}$ an orally active inhibitor of angiogenic tyrosine kinases (including the VEGF receptors 1, 2, and 3), as well as several other membrane-bound and intracellular kinases were recently approved for $\mathrm{mCRC}$ by the US Food and Drug Administration (FDA). ${ }^{24}$ Among these targeted agents, only bevacizumab and cetuximab have been approved by the Chinese FDA so far. However, because public health insurance does not cover either bevacizumab or cetuximab, most Chinese patients do not choose targeted therapy with cytotoxic drugs as first-line chemotherapy.

\section{Mechanistic and pharmacological aspects of capecitabine}

Capecitabine is a novel oral fluoropyrimidine carbamate designed to generate 5-FU selectively in tumor tissue through exploitation of higher intratumoral concentrations of thymidine phosphorylase. ${ }^{12}$ Oral capecitabine rapidly and extensively passes through the gastrointestinal tract, with a time to reach peak concentration of 2 hours and peak plasma drug concentration of 3-4 mg/L. ${ }^{25}$ It has a relatively short elimination half-life of 0.55 to 0.89 hour, ${ }^{25}$ linear pharmacokinetics, and 40\%-45\% absolute bioavailability. ${ }^{26}$

Capecitabine generates 5-FU through a three-step enzymatic cascade (Figure 1). ${ }^{27}$ It is converted first by carboxylesterase to $5^{\prime}$-deoxy-5-fluorocytidine in the liver, second by cytidine (Cyd) deaminase to $5^{\prime}$-deoxy-5-fluorouridine in the liver and tumor tissues, and finally by thymidine phosphorylase (dThdPase) to 5-FU in tumors. Carboxylesterase was found to be almost exclusively located in the liver and hepatoma, but not in other tumors and normal tissue adjacent to the tumors. Cyd deaminase was located in high concentrations in the liver and various types of solid tumors. The final enzyme dThdPase showed higher concentrations in various tumor types, including colorectal, breast, and gastric cancers, than in normal tissues. ${ }^{27}$ In primary colorectal tumors, capecitabine was preferentially activated in the tumor, with the average concentration of 5-FU being 3.2 times higher than in adjacent healthy tissue $(P=0.002) .{ }^{28}$ However, this tissue 5-FU concentration differential was not detected in liver (metastasis/ healthy tissue 5-FU concentration ratio $=1.4, P=0.49$ ). Repeated administration of capecitabine did not cause significant plasma accumulation of fluorouracil precursors 5'-deoxy-5-fluorocytidine and 5'-deoxy-5-fluorouridine. ${ }^{25}$ During long-term administration, plasma concentrations of fluorouracil increase by $10 \%-60 \%$, which is assumed not to be clinically relevant. ${ }^{25}$ Catalytic inactivation of 5-FU is performed by dihydropyrimidine dehydrogenase, which is polymorphically expressed. ${ }^{26}$

\section{Methods}

PubMed and Wanfang were searched for published clinical studies, and proceedings from the American Society of Clinical Oncology and the European Society for Medical 


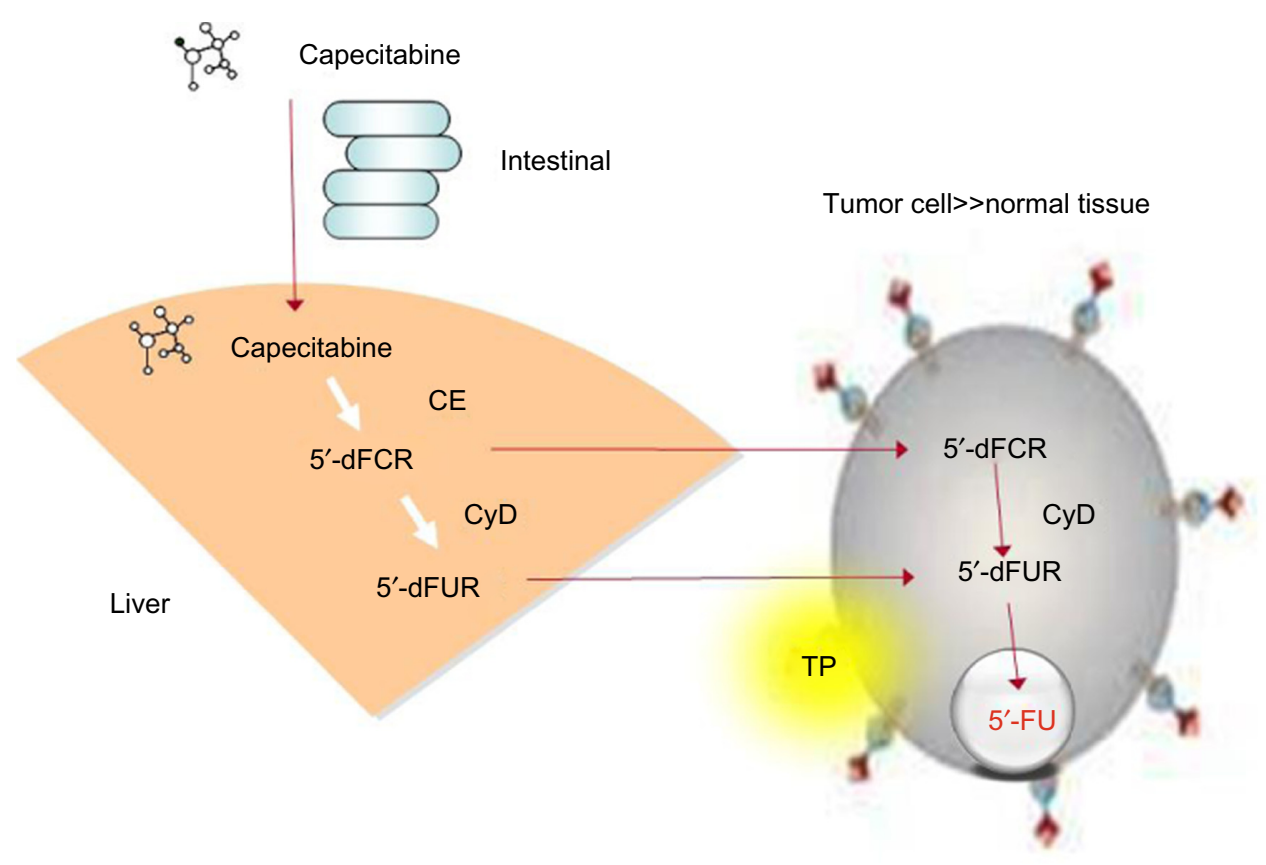

Figure I Capecitabine generates 5-fluorouracil (5-FU) through a three-step enzymatic cascade. Capecitabine is first converted to $5^{\prime}$-deoxy-5-fluorocytidine(5'-dFCR) by carboxylesterase (CE) in the liver, then by cytidine deaminase (CyD) to $5^{\prime}$-deoxy-5-fluorouridine $\left(5^{\prime}\right.$-dFUR) in the liver and tumor tissues, and finally by thymidine phosphorylase (TP) to 5-FU in tumors. Catalytic inactivation of 5-FU is done by dihydropyrimidine dehydrogenase (DPD).

Oncology were searched for abstracts pertaining to capecitabine as treatment of mCRC. Efficacy and safety data were used when possible. Missing data were designated as "not available."

\section{Capecitabine in first-line chemotherapy for $\mathrm{MCRC}$}

In the PRC, the first registered Phase II multicenter clinical trial of capecitabine in $\mathrm{mCRC}$ was done in 2004. This trial was designed to evaluate the efficacy and safety of capecitabine as first-line therapy in Chinese patients with mCRC (Table 1). ${ }^{29}$ Sixty patients with advanced CRC were recruited to receive single-agent capecitabine treatment from December 2000 to November 2001. The dosage of capecitabine was $1,250 \mathrm{mg} /$ $\mathrm{m}^{2}$ twice daily on days 1-14 of each 21-day cycle. The overall response rate (ORR) was $23.3 \%$, with $40 \%$ stable disease (SD). The median TTP and OS were 10.1 and 14.7 months, respectively, while the 1-year and 2-year survival rates were $63.9 \%$ and $33.4 \%$ respectively. As shown in Table 1, grade 3-4 adverse effects were diarrhea (6.6\%), anemia (3.3\%), and hand-foot syndrome (HFS; 1.7\%).

Based on these promising findings and results from two randomized international Phase III clinical trials, ${ }^{30,31}$ the Chinese FDA has approved capecitabine for use as a first-line therapy in patients with mCRC. Followed by this approval, combinations of capecitabine with oxaliplatin or irinotecan as first-line therapy have been investigated for treating Chinese patients with mCRC. Since oxaliplatin was approved earlier than irinotecan in the PRC, it has been used more widely in first-line chemotherapy of $\mathrm{mCRC}$, thus its combination with capecitabine has been tested in multiple studies. In one Phase II single-arm clinical trial, Li et al treated $\mathrm{mCRC}$ patients with XELOX as first-line therapy. ${ }^{32}$ Oral capecitabine $1,000 \mathrm{mg} / \mathrm{m}^{2}$ twice daily for 2 weeks and intravenous oxaliplatin $130 \mathrm{mg} /$ $\mathrm{m}^{2}$ on day 1 of each 21-day cycle was administered. The therapy was active, yielding an ORR of $49.1 \%$ and SD rate of $32.3 \%$. The median PFS and OS were 8 and 20 months, respectively. The most common grade 3-4 toxicities were neutropenia (5.6\%), nausea, and vomiting (4\%), thrombocytopenia $(2.4 \%)$, diarrhea $(2.4 \%)$, and HFS $(2.4 \%)$. The authors concluded that XELOX is a highly effective first-line treatment for Chinese patients with $\mathrm{mCRC}$. The efficacy and safety of XELOX have been compared with FOLFOX as first-line treatment for patients with $\mathrm{mCRC}$ (Table 1). In another Phase II trial, 64 patients diagnosed with mCRC were randomized into a XELOX group (oxaliplatin $85 \mathrm{mg}$ / $\mathrm{m}^{2}$ intravenously on day 1 and capecitabine $1,000 \mathrm{mg} / \mathrm{m}^{2}$ orally days $1-14$, of each 21-day cycle) or a FOLFOX group (oxaliplatin $85 \mathrm{mg} / \mathrm{m}^{2}$ intravenously on day 1 , CF $200 \mathrm{mg} / \mathrm{m}^{2}$ intravenously days $1-5,5-\mathrm{FU} 300 \mathrm{mg} / \mathrm{m}^{2}$ intravenously days 1-5, of each 21-day cycle). ${ }^{33}$ The XELOX group appeared to have a significantly higher ORR (62.5\% vs $34.4 \%)$ and grade 3-4 toxicities, including nausea and vomiting (31.1\% vs $3.1 \%)$ and neuronsensory toxicity ( $9.4 \%$ vs $0 \%)$, than the 
Table I Capecitabine in first-line treatment of Chinese patients with metastatic colorectal cancer

\begin{tabular}{|c|c|c|c|c|c|c|}
\hline Study & $\begin{array}{l}\text { Evidence } \\
\text { level }\end{array}$ & $\begin{array}{l}\text { Median } \\
\text { age (years) }\end{array}$ & $\mathbf{n}$ & Treatment detail & ORR & DCR \\
\hline Guan et $\mathrm{a}^{29}$ & Ilb & 56 & 60 & Capecitabine $\mathrm{I}, 250 \mathrm{mg} / \mathrm{m}^{2}$ bid DI-14 & $23.3 \%$ & $63.3 \%$ \\
\hline Li et $\mathrm{al}^{32}$ & Ilb & 52 & 124 & $\begin{array}{l}\text { XELOX: capecitabine } \mathrm{I}, 000 \mathrm{mg} / \mathrm{m}^{2} \text { bid D I- } 14 \text {, oxaliplatin } 85 \mathrm{mg} / \mathrm{m}^{2} \mathrm{DI} \text {, } \\
\text { every } 3 \text { weeks }\end{array}$ & $49.1 \%$ & $81.4 \%$ \\
\hline \multirow[t]{2}{*}{$\begin{array}{l}\text { Wu and } \\
\mathrm{Wu}^{70}\end{array}$} & Ilb & & 43 & $\begin{array}{l}\text { XELOX: capecitabine } 1,000 \mathrm{mg} / \mathrm{m}^{2} \text { bid DI-14, oxaliplatin } 130 \mathrm{mg} / \mathrm{m}^{2} \mathrm{DI} \text {, } \\
\text { every } 3 \text { weeks }\end{array}$ & $65.1 \%$ & $93.0 \%$ \\
\hline & & & 40 & OLF: 5 -FU I,000 mg/m² DI-2, oxaliplatin $130 \mathrm{mg} / \mathrm{m}^{2}$ DI, every 2 weeks & $55 \%$ & $95 \%$ \\
\hline \multirow[t]{2}{*}{$\begin{array}{l}\text { Luo and } \\
\text { Ouyang }^{71}\end{array}$} & Ilb & 61 & 31 & $\begin{array}{l}\text { XELOX: capecitabine } 1,000 \mathrm{mg} / \mathrm{m}^{2} \text { bid DI-14, oxaliplatin } 130 \mathrm{mg} / \mathrm{m}^{2} \mathrm{DI} \text {, } \\
\text { every } 3 \text { weeks }\end{array}$ & $48.4 \%$ & $83.9 \%$ \\
\hline & & 59 & 31 & $\begin{array}{l}\text { FOLFOX: 5-FU 2,400 mg/m² } 48 \text { hours civ, } 5 \text {-FU } 400 \mathrm{mg} / \mathrm{m}^{2} \mathrm{IV} \text { DI, } \\
\text { LV } 200 \mathrm{mg} / \mathrm{m}^{2} \mathrm{DI} \text {, oxaliplatin } 100 \mathrm{mg} / \mathrm{m}^{2} / \mathrm{DI} \text {, every } 2 \text { weeks }\end{array}$ & $51.6 \%$ & $87.1 \%$ \\
\hline \multirow[t]{2}{*}{ Deng et $\mathrm{al}^{33}$} & $\mathrm{Ilb}$ & 56 & 32 & $\begin{array}{l}\text { XELOX: capecitabine } \mathrm{I}, 000 \mathrm{mg} / \mathrm{m}^{2} \text { bid DI- } 14 \text {, oxaliplatin } 85 \mathrm{mg} / \mathrm{m}^{2} \mathrm{DI} \text {, } \\
\text { every } 3 \text { weeks }\end{array}$ & $62.5 \%$ & $84.3 \%$ \\
\hline & & 58 & 32 & $\begin{array}{l}\text { FOLFOX: } 5 \text {-FU } 300 \mathrm{mg} / \mathrm{m}^{2} \text { IV drip DI-5, LV } 200 \mathrm{mg} / \mathrm{m}^{2} \mathrm{DI}-5 \\
\text { oxaliplatin } 85 \mathrm{mg} / \mathrm{m}^{2} \mathrm{DI} \text {, every } 3 \text { weeks }\end{array}$ & $34.4 \%$ & $71.8 \%$ \\
\hline \multirow[t]{2}{*}{ Wu and $\mathrm{Sun}^{34}$} & III & 55 & 20 & $\begin{array}{l}\text { XELOX: capecitabine } 1,000 \mathrm{mg} / \mathrm{m}^{2} \text { bid DI-14, oxaliplatin } 130 \mathrm{mg} / \mathrm{m}^{2} \mathrm{DI} \text {, } \\
\text { every } 3 \text { weeks }\end{array}$ & $45.0 \%$ & $65.0 \%$ \\
\hline & & 53 & 23 & 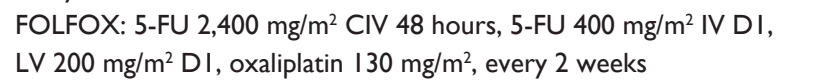 & $43.5 \%$ & $67.9 \%$ \\
\hline \multirow[t]{2}{*}{ Zhang et a ${ }^{35}$} & Ilb & & 31 & $\begin{array}{l}\text { XELOX: capecitabine } 1,000 \mathrm{mg} / \mathrm{m}^{2} \text { bid DI-14, oxaliplatin } 130 \mathrm{mg} / \mathrm{m}^{2} \mathrm{DI} \text {, } \\
\text { every } 3 \text { weeks }\end{array}$ & $48.4 \%$ & $80.6 \%$ \\
\hline & & & 67 & $\begin{array}{l}\text { FOLFOX: 5-FU 2,400-3,000 mg/m² CIV } 48 \text { hours, } 5 \text {-FU } 400 \mathrm{mg} / \mathrm{m}^{2} \text { IV DI, } \\
\text { LV } 300 \mathrm{mg} / \mathrm{m}^{2} \text { DI, oxaliplatin } 100 \mathrm{mg} / \mathrm{m}^{2} \text {, every } 2 \text { weeks }\end{array}$ & $47.8 \%$ & $80.6 \%$ \\
\hline \multirow[t]{2}{*}{ Wang et a ${ }^{36}$} & Ilb & & 38 & XELOX: capecitabine $1,000 \mathrm{mg} / \mathrm{m}^{2}$ bid DI- 14 , oxaliplatin $130 \mathrm{mg} / \mathrm{m}^{2} \mathrm{DI}$ & $47.4 \%$ & $76.3 \%$ \\
\hline & & & 46 & $\begin{array}{l}\text { mFOLFOX6: oxaliplatin } 85 \mathrm{mg} / \mathrm{m}^{2} \mathrm{DI}, \mathrm{LV} 400 \mathrm{mg} / \mathrm{m}^{2} \mathrm{DI} \\
\text { 5-FU } 400 \mathrm{mg} / \mathrm{m}^{2} \mathrm{DI}, 5 \text {-FU } 2.4 \mathrm{~g} / \mathrm{m}^{2} \mathrm{CIV} 46 \text { hours, every } 2 \text { weeks }\end{array}$ & $52.5 \%$ & $78.3 \%$ \\
\hline Choi et $\mathrm{al}^{37}$ & Ilb & 60 & 43 & $\begin{array}{l}\text { XELIRI: capecitabine } 850 \mathrm{mg} / \mathrm{m}^{2} \text { bid DI-5 every } 7 \text { days, irinotecan } \\
180 \mathrm{mg} / \mathrm{m}^{2} \mathrm{DI} \text {, every } 2 \text { weeks }\end{array}$ & $51.4 \%$ & $78.0 \%$ \\
\hline \multirow[t]{2}{*}{ Yue and $\mathrm{Liu}^{72}$} & Ilb & 47 & 39 & $\begin{array}{l}\text { XELIRI: capecitabine } 1,000 \mathrm{mg} / \mathrm{m}^{2} \text { bid DI- } 14 \text {, irinotecan } 100 \mathrm{mg} / \mathrm{m}^{2} \mathrm{DI} \text {, } \\
\text { every } 3 \text { weeks }\end{array}$ & $48.7 \%$ & $92.3 \%$ \\
\hline & & & 38 & $\begin{array}{l}\text { 5-FU } 400 \text { mg/m² DI-2, 5-FU } 600 \text { mg/m² D2 CIV, LV } 200 \text { mg DI-2, } \\
\text { every } 3 \text { weeks }\end{array}$ & $21.1 \%$ & $71.1 \%$ \\
\hline \multirow[t]{2}{*}{ Yu and $W^{38}$} & III & NA & 27 & $\begin{array}{l}\text { XELIRI: capecitabine } 625 \mathrm{mg} / \mathrm{m}^{2} \text { bid DI-14, irinotecan } 90-125 \mathrm{mg} / \mathrm{m}^{2} \mathrm{DI} \text {, } \\
\text { every } 2 \text { weeks }\end{array}$ & $51.9 \%$ & $84.6 \%$ \\
\hline & & & 16 & $\begin{array}{l}\text { FOLFIRI: CPT-II } 90-125 \mathrm{mg} / \mathrm{m}^{2} \mathrm{DI}, \mathrm{LV} 30 \mathrm{mg} / \mathrm{m}^{2} \mathrm{DI}, 5-\mathrm{FU} \\
425 \mathrm{mg} / \mathrm{m}^{2} \mathrm{DI} \text {, every } 2 \text { weeks }\end{array}$ & $31.3 \%$ & $62.5 \%$ \\
\hline Zhao et $\mathrm{a}^{73}$ & III & 61 & 48 & $\begin{array}{l}\text { XELIRI: capecitabine I,000 mg/m² bid D2-15, irinotecan } 240 \mathrm{mg} / \mathrm{m}^{2} \mathrm{DI} \text {, } \\
\text { every } 3 \text { weeks, followed by surgery if possible }\end{array}$ & $56.3 \%$ & $85.5 \%$ \\
\hline Lv and & llb & 63 & 27 & XELOX + Bev: capecitabine $1,000 \mathrm{mg} / \mathrm{m}^{2}$ bid DI-I4, oxaliplatin & $74.0 \%$ & $85.0 \%$ \\
\hline \multirow[t]{2}{*}{ Feng ${ }^{42}$} & & & & $130 \mathrm{mg} / \mathrm{m}^{2} \mathrm{DI}$, bevacizumab 5 mg/kg DI, DI5, every 3 weeks & & \\
\hline & & 64 & 26 & $\begin{array}{l}\text { XELOX: capecitabine } 1,000 \mathrm{mg} / \mathrm{m}^{2} \text { bid DI-14, oxaliplatin } 130 \mathrm{mg} / \mathrm{m}^{2} \mathrm{DI} \text {, } \\
\text { every } 3 \text { weeks }\end{array}$ & $42.0 \%$ & $62.3 \%$ \\
\hline \multirow[t]{2}{*}{$\begin{array}{l}\text { Jing and } \\
\text { Guo }\end{array}$} & $\mathrm{llb}$ & 58 & 31 & $\begin{array}{l}\text { XELOX + bevacizumab: capecitabine } 1,000 \mathrm{mg} / \mathrm{m}^{2} \text { bid DI-I4, } \\
\text { oxaliplatin } 130 \mathrm{mg} / \mathrm{m}^{2} \text { DI, bevacizumab } 7.5 \mathrm{mg} / \mathrm{kg} \text { DI, every } 3 \text { weeks }\end{array}$ & $61 \%$ & $80 \%$ \\
\hline & & 57 & 32 & $\begin{array}{l}\text { XELOX: capecitabine } 1,000 \mathrm{mg} / \mathrm{m}^{2} \text { bid DI- } 14 \text {, oxaliplatin } 130 \mathrm{mg} / \mathrm{m}^{2} \mathrm{DI} \text {, } \\
\text { every } 3 \text { weeks }\end{array}$ & $34 \%$ & $66 \%$ \\
\hline \multirow[t]{2}{*}{ Li et al $\left.\right|^{54}$} & lla & 61 & 32 & Capecitabine for maintenance: $1,000 \mathrm{mg} / \mathrm{m}^{2}$ bid DI- 14 , every 3 weeks & $33.3 \%$ & \\
\hline & & 57 & 52 & Nonmaintenance & $32.7 \%$ & \\
\hline Yang et al ${ }^{55}$ & III & $<75$ & 29 & Capecitabine for maintenance: $1,250 \mathrm{mg} / \mathrm{m}^{2}$ bid DI-14, every 3 weeks & $41.5 \%$ & $100 \%$ \\
\hline \multirow[t]{2}{*}{$\begin{array}{l}\text { Ling and } \\
\text { Zhao }^{56}\end{array}$} & III & 56 & 33 & $\begin{array}{l}\text { XELOX: capecitabine } 1,000 \mathrm{mg} / \mathrm{m}^{2} \text { bid DI-14, oxaliplatin } 130 \mathrm{mg} / \mathrm{m}^{2} \mathrm{DI} \text {, } \\
\text { every } 3 \text { weeks }\end{array}$ & $33.3 \%$ & $54.6 \%$ \\
\hline & & 54 & 33 & $\begin{array}{l}\text { XELOX + Kanglaite: capecitabine } 1,000 \mathrm{mg} / \mathrm{m}^{2} \text { bid DI-14 + oxaliplatin } \\
130 \mathrm{mg} / \mathrm{m}^{2} \mathrm{DI} \text {, Kanglaite } 100 \mathrm{~mL} \mathrm{DI}-14 \text {, every } 3 \text { weeks }\end{array}$ & $57.6 \%$ & $78.8 \%$ \\
\hline
\end{tabular}

Note: according to an oxaliplatin-specific scale.

Abbreviations: bid, bis in die (twice daily); D, day(s); CR, complete response; CPT-II, camptothecin-II; PR, partial response; NA, not available; ORR, objective response rate; DCR, disease-control rate; mTTP, median time to progression; mPFS, median progression-free survival; HFS, hand-foot syndrome; CIV, continuous intravenous; 5 -FU, 5-fluorouracil; OS, overall survival; LV, leucovorin. 


\begin{tabular}{|c|c|c|c|c|c|c|c|}
\hline \multirow{2}{*}{$\begin{array}{l}\text { mTTPI } \\
\text { mPFS }\end{array}$} & \multirow{2}{*}{$\begin{array}{l}\text { Median } \\
\text { OS }\end{array}$} & \multicolumn{6}{|c|}{ Grade 3-4 adverse effects } \\
\hline & & $\begin{array}{l}\text { Diarrhea } \\
\text { (\%) }\end{array}$ & $\begin{array}{l}\text { Vomiting/ } \\
\text { nausea }(\%)\end{array}$ & $\begin{array}{l}\text { Neutropenia/ } \\
\text { leukopenia (\%) }\end{array}$ & $\begin{array}{l}\text { Anemia } \\
(\%)\end{array}$ & $\begin{array}{l}\text { Neuropathy } \\
\text { (\%) }\end{array}$ & $\begin{array}{l}\text { HFS } \\
(\%)\end{array}$ \\
\hline 10.1 & 14.7 & 6.7 & 0 & 0 & 0 & 0 & 1.7 \\
\hline 8.0 & 20.0 & 15.3 & 35.4 & 50.8 & 4 & 42 & 25.0 \\
\hline NA & NA & 0 & 2.3 & 14 & 0 & 4.7 & 0 \\
\hline NA & NA & 2.5 & 2.5 & 17.5 & 5 & 7.5 & 0 \\
\hline 6.8 & NA & 16.1 & 58.1 & 67.7 & NA & 6.5 & 48.4 \\
\hline 7.1 & NA & 12.9 & 67.8 & 83.9 & NA & 22.6 & 0.0 \\
\hline NA & NA & NA & 31.3 & 6.3 & 0 & 9.4 & NA \\
\hline NA & NA & NA & 3.1 & 6.3 & 0 & 0 & NA \\
\hline 7.0 & 13.1 & 0 & 5 & 10 & 0 & 5 & 10 \\
\hline 7.0 & 13.8 & 0 & 8.7 & 30.4 & 0 & 4.3 & 0 \\
\hline 7.1 & 13.7 & 3.2 & 9.7 & 0 & 0 & 0 & 9.7 \\
\hline 7.5 & $14 . \mid$ & 4.5 & 13.4 & 16.4 & 0 & 0 & 0 \\
\hline NA & NA & 2.2 & 13.4 & 19.8 & 19.4 & 15.9 & 25 \\
\hline NA & NA & 0 & 12.2 & 16.8 & 17.3 & 16.4 & 0.2 \\
\hline 10 & 15.4 & 8.1 & 0 & 5.4 & 2.7 & NA & 2.7 \\
\hline NA & NA & 12.8 & 2.6 & 10.3 & 0 & NA & NA \\
\hline NA & NA & 0 & 2.6 & 5.3 & 0 & NA & NA \\
\hline 12.5 & 17.9 & 7.4 & 0 & 0 & NA & 22.2 & 7.4 \\
\hline 8.4 & 14.2 & 18.8 & 6.3 & 0 & NA & 0 & 0 \\
\hline 16.7 & 27.5 & 17 & 4.3 & 8.5 & 0 & 0 & 4.3 \\
\hline 7.3 & NA & 0 & 0 & 3.7 & 0 & 0 & 0 \\
\hline 5.4 & NA & 0 & 0 & 7.7 & 0 & 0 & 0 \\
\hline NA & NA & 23.0 & NA & 35.0 & NA & 10.0 & 10.0 \\
\hline NA & NA & 25.0 & NA & 31.0 & NA & 13.0 & 6.0 \\
\hline 9 & 40.4 & $3 \%$ & 0 & 0 & 0 & 0 & 0 \\
\hline 6.5 & 21.5 & 0 & 0 & 0 & 0 & 0 & 0 \\
\hline 12.4 & NA & 0 & 0 & 0 & 0 & 0 & 12.0 \\
\hline NA & NA & NA & 0 & 6.1 & 9.1 & 9.1 & 6.1 \\
\hline NA & NA & NA & 0 & 3 & 0 & 6.1 & 3 \\
\hline
\end{tabular}


FOLFOX group. While most studies showed similar ORR and PFS or OS, there was significantly more grade 3-4 HFS in XELOX than FOLFOX..$^{34-36}$

The efficacy and toxicity of XELIRI in mCRC was evaluated by a Phase II dual-center clinical trial. ${ }^{37}$ Forty-three patients were enrolled between 2004 and 2005 in Hong Kong. Camptothecin-11 was administered intravenously at a dose of $180 \mathrm{mg} / \mathrm{m}^{2}$ on day 1 and capecitabine was given orally at a dose of $850 \mathrm{mg} / \mathrm{m}^{2}$ twice daily for 5 days, followed by 2 days of rest every 7 days in a 14-day cycle. The ORR was $51.4 \%$ and the SD rate was $27 \%$. The median OS was 15.4 months, and the 1 -year survival rate was $75.3 \%$. The most common grade 3 toxicities were neutropenia $(5.4 \%)$, diarrhea $(8.1 \%)$, and HFS (2.7\%). Yu and Wu treated 43 patients with mCRC with first-line XELIRI or FOLFIRI. ${ }^{38}$ XELIRI tended to have a much higher ORR (51.9\% vs 31.3\%), TTP (12.5 vs 8.4 months), and OS (17.9 vs 14.2 months) than FOLFIRI. ${ }^{38}$ In two Phase II studies, Chinese patients appeared to tolerate XELIRI well, with low incidence of grade 3-4 adverse effects. No drug-related death was reported in Chinese patients. The variances in tolerability of irinotecan between Chinese and Caucasians may probably be due to UGT1A1 genotype differences. The frequency of $U G T 1 A 1^{*} 6$ or $U G T 1 A 1 * 28$ gene polymorphisms is much lower in Chinese than in Caucasians. ${ }^{39,40}$

In a Phase II clinical study, Liu and Zhang treated 112 Chinese patients with mCRC with first-line XELOX or XELIRI to compare efficacy and toxicities. ${ }^{41}$ No statistical differences were observed in ORR $(27.1 \%$ vs $25 \%)$ or median PFS (6.2 vs 7.1 months) between the two groups. Grade 3-4 diarrhea appeared substantially more commonly in the XELIRI group $(27.5 \%$ vs $2.1 \%, P=0.002)$. However, fewer patients treated with XELIRI developed grade 3-4 neurotoxicity than with XELOX (2.5\% vs $22.9 \%$, $P=0.018)$.

Capecitabine has also been combined with monoclonal antibodies and other biological agents in Chinese patients with mCRC. XELOX and bevacizumab have been shown to exhibit higher response than XELOX. Lv and Feng investigated the efficacy and toxicity of bevacizumab $(5 \mathrm{mg} / \mathrm{kg}$, day 1 , day 15) plus XELOX (oxaliplatin $130 \mathrm{mg} / \mathrm{m}^{2}$ intravenously day 1 , plus capecitabine $1,000 \mathrm{mg} / \mathrm{m}^{2}$ orally twice daily, days $1-14$, every 21 days) on patients with mCRC. ${ }^{42}$ Fifty-three patients were randomized to receive either XELOX or XELOX plus bevacizumab. The patients treated with XELOX plus bevacizumab achieved remarkably higher complete response (CR; 15\% vs 4\%) and partial response (PR; 59\% vs 38\%) rates than XELOX alone. Median TTP was 7.3 months in the combination group, compared with 5.4 months in XELOX group. No significant difference of adverse effects was observed between the two groups. In a retrospective study, the effect of XELOX plus bevacizumab was compared to XELOX alone in CRC patients with potential resectable live metastasis. ${ }^{43}$ A total of 63 patients were divided into two groups to receive oxaliplatin $\left(130 \mathrm{mg} / \mathrm{m}^{2}\right)$ and capecitabine $\left(1,000 \mathrm{mg} / \mathrm{m}^{2}\right)$ with or without bevacizumab $(7.5 \mathrm{mg} / \mathrm{kg})$. The XELOX plus bevacizumab group had a significantly higher curative (R0) resection rate (45\% vs 19\%) and ORR (61\% vs 34\%). Grade 3-4 adverse effects, including neutropenia and diarrhea, were comparable between the two groups.

A Phase II clinical trial for mCRC treated patients with first-line capecitabine and cetuximab. ${ }^{44}$ Forty elderly patients (>65 years) whose Kirsten rat sarcoma (KRAS) had been tested were randomly separated into two groups to receive XELOX with or without cetuximab. Oxaliplatin $\left(130 \mathrm{mg} / \mathrm{m}^{2}\right)$ was administered intravenously on day 1 , and capecitabine $\left(1,000 \mathrm{mg} / \mathrm{m}^{2}\right)$ was given orally twice a day, days $1-14$, on a 3-week cycle. The treatment group, where all patients had wild-type KRAS, was given a loading dose of cetuximab $400 \mathrm{mg} / \mathrm{m}^{2}$ the first time, followed by $250 \mathrm{mg} / \mathrm{m}^{2}$ intravenously on day 1 of each cycle. Compared with the control group, the treatment group had significantly higher ORR $(12 \%$ vs $7 \%, P<0.05)$ and TTP ( 7.7 vs 5.4 months). Grade 1-2 skin rash was observed in $55 \%$ of the patients in the treatment group.

Endostatin, a carboxyl-terminal fragment digested from collagen XVIII, which exhibits inhibitive effects on endothelial cell migration, proliferation, and angiogenesis, ${ }^{45}$ was approved for non-small-cell lung cancer in the PRC. ${ }^{46}$ Fan et al evaluated the efficacy and safety of endostatin combined with XELIRI in patients with mCRC. ${ }^{47}$ Median PFS and OS were 6.2 months and 13.7 months, respectively, with an ORR of $46.4 \%$. The most frequent grade 3-4 adverse effects were neutropenia (39.3\%), anemia (35.7\%), stomatitis $(14.3 \%)$, thrombocytopenia $(17.9 \%)$, and diarrhea (10.7\%).

Thalidomide, a sedative and anti-inflammatory drug used in immunotherapy, has been recently explored as an antineoplastic agent, possibly due to its dual effect on immunomodulation and inhibition of angiogenesis. ${ }^{48-50} \mathrm{Zhou}$ et al reported that a combination of oral capecitabine $\left(1,250 / \mathrm{m}^{2}\right.$ twice daily, days 1-14) with oral thalidomide (100 mg, days 1-21) in $\mathrm{mCRC}$ patients led to a high response rate (CR 8.33\%, PR 38.88\%) and SD rate (30.56\%). ${ }^{51}$ The regimen was well tolerated with all grade toxicities, including neurotoxicity 
(58.33\%), constipation (52.77\%), and HFS (44.44\%). Chen and Lou further investigated the effect of combined thalidomide (200 mg daily, days 1-10) with XELOX (infusional oxaliplatin $130 \mathrm{mg} / \mathrm{m}^{2}$ day 1 , oral capecitabine $1,000 \mathrm{mg} / \mathrm{m}^{2}$ twice a day, days 1-14) in mCRC. ${ }^{52}$ A total of $4.75 \%$ of patients achieved CR, $47.62 \% \mathrm{PR}, 23.81 \% \mathrm{SD}$, and $23.81 \%$ PD. The median TTP and OS were 7.2 and 13.6 months, respectively. Major adverse effects included neurotoxicity, leucopenia, and HFS. A randomized controlled Phase II trial was carried out to evaluate the efficacy and safety of the combination of XELOX with thalidomide as first-line treatment of mCRC. ${ }^{53}$ Eighty-nine patients with mCRC were randomly divided into treatment group (thalidomide with XELOX) or control group (XELOX). The primary end point, PFS (5.6 vs 5.2 months, $P=0.307$ ), and one secondary end point, ORR ( $34.1 \%$ vs $26.7 \%, P=0.446)$, were similar between the two groups. However, another secondary endpoint, disease-control rate (DCR), significantly improved $(63.6 \%$ vs $42.2 \%, P=0.043)$ in the treatment group. Patients treated with thalidomide experienced more grade 3-4 constipation $(20.5 \%$ vs $4.4 \%, P=0.022)$, which did not result in treatment interruption. The rate of lethargy increased, but had no statistical significance $(13.6 \%$ vs $4.4 \%, P=0.13)$.

The role of capecitabine as maintenance therapy after first-line or second-line treatment for mCRC has been investigated in three Phase II clinical trials. Li et al divided patients with $\mathrm{mCRC}$ who had achieved response from firstline chemotherapy into a capecitabine-maintenance group $\left(1,000 \mathrm{mg} / \mathrm{m}^{2}\right.$ twice daily on days $1-14$, every 21 days $)$ and a nonmaintenance group. ${ }^{54}$ Patients in the maintenance group showed significantly longer TTP (9 vs 6.5 months) and OS (40.4 vs 21.5 months) than those in the nonmaintenance group. Yang et al treated $35 \mathrm{mCRC}$ patients who received 5-FU-based first-line or second-line chemotherapy with capecitabine $\left(1,000-1,250 \mathrm{mg} / \mathrm{m}^{2}\right.$, days $1-14$, every 3 weeks) as maintenance therapy. ${ }^{55}$ The total median PFS was 12.2 months, with 12.4 months for first-line and 11.6 months for second-line treatment. Only grade 3-4 HFS $(12 \%)$ was observed. In another study mentioned previously, Li et al treated $124 \mathrm{mCRC}$ patients with XELOX as first-line chemotherapy. ${ }^{32}$ Among the patients who achieved objective response or SD after six cycles of XELOX, 22 patients chose to continue oral capecitabine as maintenance therapy until disease progression. These patients had a significantly longer duration of disease control than those without maintenance (14 vs 9 months, $P=0.041$ ).

Several studies have combined capecitabine with Chinese herbal medicine or natural products. Ling and Zhao reported on a combination of oxaliplatin, capecitabine, and Coix seed oil (Zhejiang Kanglaite Pharmaceutical Co., Hanzhou, Zhejiang, People's Republic of China) injection in mCRC. ${ }^{56}$ Sixty-four patients were treated by either XELOX with or without Kanglaite (10 g, days $1-14$, every 2 weeks). The patients who were treated with Kanglaite showed significantly higher ORR (57.6\% vs $33.3 \%, P=0.048)$, improved quality of life $(P<0.01)$, and fewer adverse effects than the control group.

\section{Capecitabine in second-line treatment or beyond for MCRC}

Although a Phase II study from the MD Anderson Cancer Center showed that capecitabine achieved no response in patients with $\mathrm{mCRC}$ who had progressed on previous 5-FU therapy, ${ }^{57}$ a few Chinese studies have demonstrated efficacy of capecitabine in $\mathrm{mCRC}$ patients refractory to 5-FU (Table 2). In one study, 47 patients with $\mathrm{mCRC}$ who had been resistant to 5-FU were treated with capecitabine $\left(1,250 \mathrm{mg} / \mathrm{m}^{2}\right.$ twice daily, days $1-14$, every 3 weeks): ${ }^{58} 17 \%$ of patients who achieved response had a median TTP of 8.5 months, $44.7 \%$ of patients with SD had a median TTP of 4.8 months, and median OS of all patients was 10.4 months. Another study also confirmed the efficacy of capecitabine in mCRC patients who had become resistant to 5-FU. ${ }^{59}$ The median ORR and DCR were $18.8 \%$ and $65.7 \%$, respectively; the median OS was 11 months.

XELIRI as second-line treatment in mCRC patients who had been refractory to oxaliplatin was evaluated in several studies. Zhou et al reported that 38 patients with mCRC after failure of oxaliplatin were given capecitabine $\left(1,000 \mathrm{~g} / \mathrm{m}^{2}\right.$ twice daily, days 1-14) and intravenous irinotecan $\left(100 \mathrm{mg} / \mathrm{m}^{2}\right.$, day 1 , day 8 ) every 3 weeks. ${ }^{60}$ The ORR and DCR were $5.9 \%$ and $61.8 \%$, respectively, and the median TTP and OS were 4.5 and 11 months, respectively. The most common grade 3-4 adverse events were leukopenia (10.5\%), neutropenia (13.2\%), nausea and vomiting (10.5\%), and diarrhea (7.9\%). In another study, 39 patients with $\mathrm{mCRC}$ who had progressed on oxaliplatin treatment received infusional irinotecan $\left(180 \mathrm{mg} / \mathrm{m}^{2}\right.$, day 1$)$ with oral capecitabine $\left(1,000-1,250 \mathrm{mg} / \mathrm{m}^{2}\right.$ twice a day, days $1-14$, every 3 weeks). ${ }^{61}$ The ORR was $38.5 \%$, with low grade 3-4 toxicities, including diarrhea (10.3\%) and neutropenia (17.9\%). Capecitabine (1,000 mg/m² twice a day, days $1-14)$ with weekly irinotecan $\left(60 \mathrm{mg} / \mathrm{m}^{2}\right)$ also resulted in a high response $(\mathrm{ORR}=42.8 \%)$, a long median TTP (6.5 months), tolerable toxicities (grade 3-4 diarrhea [4.76\%], and neutropenia $[4.76 \%]){ }^{62}$

In eleven patients with $\mathrm{mCRC}$, the combination of bevacizumab and capecitabine was evaluated. ${ }^{63}$ Bevacizumab was given at dosages of $5 \mathrm{mg} / \mathrm{kg}$ or $10 \mathrm{mg} / \mathrm{kg}$ every 2 weeks or 


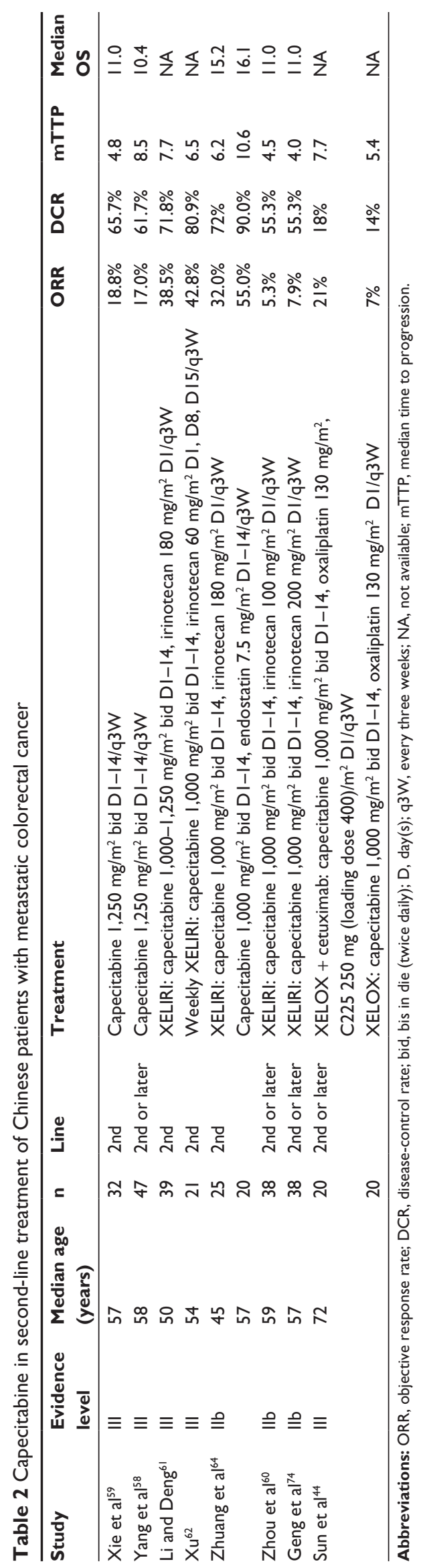

$15 \mathrm{mg} / \mathrm{kg}$ every 3 weeks. All patients received capecitabine $2,000 \mathrm{mg} / \mathrm{m}^{2}$ per day for 14 days. The DCR was $90.9 \%$, with 5 PR, 5 SD. The PFS and OS were 4 months and 15 months, respectively. Only grade $1-2$ hypertension (27.3\%), proteinuria (36.4\%), HFS (54.6\%), diarrhea (27.3\%), and neutropenia $(18.2 \%)$ were observed.

The efficacy and safety of endostatin combined with capecitabine were also tested in patients with $\mathrm{MCRC}$ who had failed first-line chemotherapy. A retrospective study compared XELIRI with capecitabine plus endostatin as second-line chemotherapy for oxaliplatin-resistant patients with mCRC ${ }^{64}$ When compared to XELIRI, capecitabine plus endostatin showed significantly higher ORR ( $55 \%$ vs $32 \%$ ), longer TTP (10.6 months vs 6.2 months), and comparable OS (16.1 months vs 15.2 months). The incidences of neutropenia and diarrhea were higher in the XELIRI group than in the endostatin group $(P<0.01)$.

A combination of gemcitabine $\left(1,000 \mathrm{mg} / \mathrm{m}^{2}\right.$, day 1 , day 8$)$ with capecitabine $\left(1,250 \mathrm{mg} / \mathrm{m}^{2}\right.$ twice daily, days 1-14) or infusional 5-FU/LV (5-FU $500 \mathrm{mg} / \mathrm{m}^{2}$, days $1-5$, LV $200 \mathrm{mg} / \mathrm{m}^{2}$, days $1-5$ ) in patients with $\mathrm{mCRC}$ who had failed at least two standard chemotherapy regimens has also been investigated. The ORR and SD rate among 19 patients treated with capecitabine and gemcitabine were $9.4 \%$ and $25 \%$, respectively. The only grade $3-4$ adverse effect was neutropenia (3.1\%). The authors concluded that the combination offers encouraging results and is well tolerated.

\section{Capecitabine in senile patients}

Wang and Zhang treated 25 elderly patients ( $\geq 60$ years) with single-agent capecitabine $1,250 \mathrm{mg} / \mathrm{m}^{2}$ twice daily from day 1 to day 14 in 3-week cycles. ${ }^{65}$ Eight patients achieved PR, and seven maintained SD. The ORR and DCR were $32 \%$ and $60 \%$, respectively. Major side effects included HFS (16\%), nausea and vomiting (16\%), diarrhea (12\%), and anorexia $(16 \%)$, most of which were minor. Zhao et al evaluated the combination of capecitabine and oxaliplatin as first-line treatment in 58 elderly patients ( $\geq 65$ years) with mCRC. ${ }^{66}$ Oxaliplatin was administered intravenously at a dose of $130 \mathrm{mg} / \mathrm{m}^{2}$ on day 1 , and capecitabine orally, $1,000 \mathrm{mg} / \mathrm{m}^{2}$ twice a day on days $1-14$ every 3 weeks. Among the patients, the ORR was $48.28 \%$, with three CR and 25 PR. The median OS was 15.8 months, and the median TTP was 8.1 months. Major toxicities, including HFS (43.1\%), neural toxicity (46.6\%), thrombocytopenia (37.9\%), neutropenia (37.9\%), and nausea and vomiting (44.8\%) were mostly grade $1-2$. The combination of capecitabine with oxaliplatin also showed 
encouraging results for senile patients with $\mathrm{mCRC}$ in several other studies. ${ }^{67,68}$

\section{Cost-effectiveness}

Compared with FOLFOX for first-line mCRC, XELOX appears to be cost-effective. In a retrospective cohort study from Hong Kong, total costs of drugs, outpatient visits, tests, and travel of 30 patients with mCRC treated with XELOX were compared with those treated with FOLFOX. ${ }^{69}$ XELOX provided superior cost-effectiveness compared with FOLFOX with respect to scheduled cost (US \$2,046 vs US \$2,152), unscheduled cost (US \$240 vs US \$421), and societal cost (US \$2,292 vs US \$4,915). The total cost for FOLFOX was $37 \%$ greater than that of XELOX. Wang et al evaluated the costs of XELOX and FOLFOX as first-line chemotherapy for $\mathrm{mCRC} .{ }^{36}$ The mean chemotherapy costs were similar between the two regimens. However, the mean hospitalization times ( 7.3 vs 9.3 times) and days ( 55 vs 88.3 days) were significantly lower in patients treated with XELOX than those treated with FOLFOX.

\section{Conclusion}

Capecitabine has been used in $\mathrm{mCRC}$ in the PRC for nearly a decade now. Despite this, there is still a lack of randomized clinical trials comparing the effectiveness of capecitabine with infusional 5-FU in the Chinese population. Most Chinese studies were not randomized, and a large portion of them were retrospective analyses. Capecitabine displayed high efficacy in Chinese patients with $\mathrm{mCRC}$ whether it was used as a single drug or in combination therapy. Chinese patients appeared to tolerate higher doses of capecitabine and showed fewer adverse effects than Caucasians. However, the specific combination and dosage regimens of chemotherapy agents for Chinese are yet to be ascertained. ${ }^{70-74}$

\section{Acknowledgments}

This study was supported by the National Natural Science Foundation of China (30672408), the Guangzhou Bureau of Science and Technology (2006Z3-E0041), and the Sun Yat-sen University 985 Program Initiation Fund (China).

\section{Disclosure}

The authors report no conflicts of interest in this work.

\section{References}

1. Parkin DM. International variation. Oncogene. 2004;23(38):6329-6340.

2. Parkin DM, Bray F, Ferlay J, Pisani P. Global cancer statistics, 2002. CA Cancer J Clin. 2005;55(2):74-108.
3. Center MM, Jemal A, Ward E. International trends in colorectal cancer incidence rates. Cancer Epidemiol Biomarkers Prev. 2009;18(6): $1688-1694$.

4. Chiu BC, Ji BT, Dai Q, et al. Dietary factors and risk of colon cancer in Shanghai, China. Cancer Epidemiol Biomarkers Prev. 2003;12(3): 201-208.

5. Xu AG, Yu ZJ, Jiang B, et al. Colorectal cancer in Guangdong Province of China: a demographic and anatomic survey. World J Gastroenterol. 2010;16(8):960-965.

6. Dai Z, Zheng RS, Zou XN, et al. [Analysis and prediction of colorectal cancer incidence trend in China]. Zhonghua Yu Fang Yi Xue Za Zhi. 2012;46(7):598-603. Chinese.

7. Li M, Gu J. Changing patterns of colorectal cancer over the recent two decades in China. Zhonghua Wei Chang Wai Ke Za Zhi. 2004;7(3): 214-217.

8. Goldberg RM. Therapy for metastatic colorectal cancer. Oncologist 2006;11(9):981-987.

9. Kelly H, Goldberg RM. Systemic therapy for metastatic colorectal cancer: current options, current evidence. J Clin Oncol. 2005;23(20): 4553-4560.

10. de Gramont A, Bosset JF, Milan C, et al. Randomized trial comparing monthly low-dose leucovorin and fluorouracil bolus with bimonthly high-dose leucovorin and fluorouracil bolus plus continuous infusion for advanced colorectal cancer: a French intergroup study. J Clin Oncol. 1997;15(2):808-815.

11. Schwarz RE, Coit DG, Groeger JS. Transcutaneously tunneled central venous lines in cancer patients: an analysis of device-related morbidity factors based on prospective data collection. Ann Surg Oncol. 2000;7(6): 441-449.

12. Van Cutsem E, Hoff PM, Harper P, et al. Oral capecitabine vs intravenous 5-fluorouracil and leucovorin: integrated efficacy data and novel analyses from two large, randomised, phase III trials. $\mathrm{Br} J$ Cancer. 2004;90(6):1190-1197.

13. Cassidy J, Clarke S, Díaz-Rubio E, et al. XELOX vs FOLFOX-4 as first-line therapy for metastatic colorectal cancer: NO16966 updated results. Br J Cancer. 2011;105(1):58-64.

14. Ducreux M, Bennouna J, Hebbar M, et al. Capecitabine plus oxaliplatin (XELOX) versus 5-fluorouracil/leucovorin plus oxaliplatin (FOLFOX-6) as first-line treatment for metastatic colorectal cancer. Int J Cancer. 2011;128(3):682-690.

15. Díaz-Rubio E, Tabernero J, Gómez-España A, et al. Phase III study of capecitabine plus oxaliplatin compared with continuous-infusion fluorouracil plus oxaliplatin as first-line therapy in metastatic colorectal cancer: final report of the Spanish Cooperative Group for the Treatment of Digestive Tumors Trial. J Clin Oncol. 2007;25(27): 4224-4230.

16. Porschen R, Arkenau HT, Kubicka S, et al. Phase III study of capecitabine plus oxaliplatin compared with fluorouracil and leucovorin plus oxaliplatin in metastatic colorectal cancer: a final report of the AIO Colorectal Study Group. J Clin Oncol. 2007;25(27):4217-4223.

17. Rothenberg ML, Cox JV, Butts C, et al. Capecitabine plus oxaliplatin (XELOX) versus 5 -fluorouracil/folinic acid plus oxaliplatin (FOLFOX-4) as second-line therapy in metastatic colorectal cancer: a randomized phase III noninferiority study. Ann Oncol. 2008;19(10): $1720-1726$.

18. Saltz LB, Clarke S, Díaz-Rubio E, et al. Bevacizumab in combination with oxaliplatin-based chemotherapy as first-line therapy in metastatic colorectal cancer: a randomized phase III study. J Clin Oncol. 2008;26(12):2013-2019.

19. Hochster HS, Hart LL, Ramanathan RK, et al. Safety and efficacy of oxaliplatin and fluoropyrimidine regimens with or without bevacizumab as first-line treatment of metastatic colorectal cancer: results of the TREE Study. J Clin Oncol. 2008;26(21):3523-3529.

20. Fuchs CS, Marshall J, Mitchell E, et al. Randomized, controlled trial of irinotecan plus infusional, bolus, or oral fluoropyrimidines in first-line treatment of metastatic colorectal cancer: results from the BICC-C Study. J Clin Oncol. 2007;25(30):4779-4786. 
21. Köhne CH, De Greve J, Hartmann JT, et al. Irinotecan combined with infusional 5-fluorouracil/folinic acid or capecitabine plus celecoxib or placebo in the first-line treatment of patients with metastatic colorectal cancer. EORTC study 40015. Ann Oncol. 2008;19(5):920-926.

22. Ciombor KK, Berlin J, Chan E. Aflibercept. Clin Cancer Res. 2013;19(8):1920-1925.

23. Grothey A, Van Cutsem E, Sobrero A, et al. Regorafenib monotherapy for previously treated metastatic colorectal cancer (CORRECT): an international, multicentre, randomised, placebo-controlled, phase 3 trial. Lancet. 2013;381(9863):303-312.

24. [No authors listed]. FDA approves aflibercept (Zaltrap) for metastatic colorectal cancer. Oncology (Williston Park). 2012;26(9):842, 873.

25. Reigner B, Blesch K, Weidekamm E. Clinical pharmacokinetics of capecitabine. Clin Pharmacokinet. 2001;40(2):85-104.

26. Schellens JH. Capecitabine. Oncologist. 2007;12(2):152-155.

27. Miwa M, Ura M, Nishida M, et al. Design of a novel oral fluoropyrimidine carbamate, capecitabine, which generates 5-fluorouracil selectively in tumours by enzymes concentrated in human liver and cancer tissue. Eur J Cancer. 1998;34(8):1274-1281.

28. Schuller J, Cassidy J, Dumont E, et al. Preferential activation of capecitabine in tumor following oral administration to colorectal cancer patients. Cancer Chemother Pharmacol. 2000;45(4):291-297.

29. Guan ZZ, Liu DG, Yu BM, et al. [First-line Xeloda (capecitabine) treatment for advanced and recurrent colorectal cancer]. Zhonghua Zhong Liu Za Zhi. 2004;26(2):119-121. Chinese.

30. Hoff PM, Ansari R, Batist G, et al. Comparison of oral capecitabine versus intravenous fluorouracil plus leucovorin as first-line treatment in 605 patients with metastatic colorectal cancer: results of a randomized phase III study. J Clin Oncol. 2001;19(8):2282-2292.

31. Van Cutsem E, Twelves C, Cassidy J, et al. Oral capecitabine compared with intravenous fluorouracil plus leucovorin in patients with metastatic colorectal cancer: results of a large phase III study. J Clin Oncol. 2001;19(21):4097-4106.

32. Li YH, Luo HY, Wang FH, et al. Phase II study of capecitabine plus oxaliplatin (XELOX) as first-line treatment and followed by maintenance of capecitabine in patients with metastatic colorectal cancer. J Cancer Res Clin Oncol. 2010;136(4):503-510.

33. Deng YJ, Liang J, Qiu ZC, Li DM. Comparison different effects between L-OHP+CAPE and FOLFOX regimen in the treatment of patients with carcinoma of colon and rectum. Med Innov China. 2011;8(15):9-11.

34. Wu FH, Sun YY. The efficacy and toxicity of XELOX regimen versus FOLFOX4 in the treatment of metastatic colorectal cancer. Clin Med Inf China. 2011;24(8):3-4. Chinese.

35. Zhang JW, Miu JH, Zhao F, Chen SB, Guo XD. Analysis of effects of the combination of capecitabine and oxaliplatin as first-line chemotherapy on metastatic colorectal cancer. Mod Med J. 2008;36(1):37-39.

36. Wang W, Lin XQ, Hu B, et al. Clinical and drug economic comparison between Xelox and mFOLFOX-6 in the treatment of advanced colorectal carcinoma. Shanghai Med Pharmacol. 2011;32(12):594-597.

37. Choi CK, Chan RT, Tung SY, et al. Efficacy of combination chemotherapy with irinotecan (CPT-11) plus capecitabine in patients with metastatic or advanced colorectal carcinoma - a dual-centre phase II study: the MAC-6. Clin Oncol (R Coll Radiol). 2008;20(2):168-175.

38. Yu BM, Wu WQ. Irinotecan combined with fluoropyrimidine in treatment for advanced/metastatic colorectal carcinoma. Zhonghua Wai Ke Za Zhi. 2005;43(9):557-560.

39. Gao J, Zhou J, Li Y, Lu M, Jia R, Shen L. UGT1A1 6/28 polymorphisms could predict irinotecan-induced severe neutropenia not diarrhea in Chinese colorectal cancer patients. Med Oncol. 2013;30(3):604.

40. Su Y, Zhang J, Zhu ZG. The relationship between the efficacy and adverse events of irinotecan-based chemotherapy and UGT1A1 gene polymorphism. Chin Clin Oncol. 2011;16(4):362-366.

41. Liu HY, Zhang B. Clinical study of oxaliplatin or irinotecan plus capecitabine in the treatment of advanced colorectal cancer. Zhongguo Lin Chuang Yao Li Xue Za Zhi. 2013;29(4):243-245.
42. Lv Q, Feng GG. Bevacizumab combined with CapeOx in treatment with colorectal cancer. Mod J Integr Tradit Chin West Med. 2011; 20(18):2213-2218. Chinese.

43. Jiang XH, Guo YJ. Bevacizumab combined with CapeOx as conversion therapy of metastatic colorectal cancer. Zhongguo Yi Shi Za Zhi. 2012;14(10):1404-1407. Chinese.

44. Sun J, Jiang LN, Pan SY. Clinical observation of 20 elderly patients with advanced colorectal cancer in the regimen of cetuximab plus XELOX. Her Med. 2012;31(1):17-19. Chinese.

45. Dhanabal M, Ramchandran R, Volk R, et al. Endostatin: yeast production, mutants, and antitumor effect in renal cell carcinoma. Cancer Res. 1999;59(1):189-197.

46. Wang J, Sun Y, Liu Y, et al. [Results of randomized, multicenter, double-blind phase III trial of rh-endostatin (YH-16) in treatment of advanced non-small cell lung cancer patients]. Zhongguo Fei Ai Za Zhi. 2005;8(4):283-290. Chinese.

47. Fan CZ, Dai H, Chu YP. The efficacy of recombinant human endostatin combined with chemotherapy in treating metastatic colorectal cancer. Cancer. 2010;30(12):1065-1068.

48. Ladetto M, Pagliano G, Ferrero S, et al. Major tumor shrinking and persistent molecular remissions after consolidation with bortezomib, thalidomide, and dexamethasone in patients with autografted myeloma. $J$ Clin Oncol. 2010;28(12):2077-2084.

49. Noman AS, Koide N, Hassan F, et al. Thalidomide inhibits lipopolysaccharide-induced tumor necrosis factor-alpha production via downregulation of MyD88 expression. Innate Immun. 2009;15(1):33-41.

50. D'Amato RJ, Loughnan MS, Flynn E, Folkman J. Thalidomide is an inhibitor of angiogenesis. Proc Natl Acad Sci U S A. 1994;91(9): 4082-4085.

51. Zhou M, Wang YL, Zhang H, Qian KQ. The treatment of capecitabine combined with thalidomide in 39 cases with advanced colorectal cancer. Chin J Cancer. 2005;14(12):837-838. Chinese.

52. Chen XF, Lou J. Thalidomide combined with XELOX in the treatment of 42 cases with advanced colorectal cancer. Clin Med. 2010;19(21):70-71.

53. Lv J, Liu N, Liu KW, Ding AP, Wang H, Qiu WS. A randomised controlled Phase II trial of the combination of XELOX with thalidomide for the first-line treatment of metastatic colorectal cancer. Cancer Biol Med. 2012;9(2):111-114.

54. Li Y, Li J, Lu M, Wang XC, Shen L. Capecitabine maintenance therapy after first-Line chemotherapy in patients with metastatic colorectal cancer. Chin J Cancer Res. 2010;22(3):181-185.

55. Yang DY, Li Y, Liu JH, Jiang WW, Ma D. Study of capecitabine maintenance therapy on metastatic colorectal patients with disease optimally controlled after chemotherapy. Pract J Med. 2013;29(8):1299-1302. Chinese.

56. Ling XL, Zhao D. Short term efficacy of oxaliplatin, capecitabine and Kanglaite in the treatment of advanced colorectal carcinoma. Med Inf. 2011;2:377-379. Chinese.

57. Hoff PM, Pazdur R, Lassere Y, et al. Phase II study of capecitabine in patients with fluorouracil-resistant metastatic colorectal carcinoma. J Clin Oncol. 2004;22(11):2078-2083.

58. Yang WD, Li B, Wang BC. Efficacy of capecitabine in patients with 5-FU-resistant metastatic colorectal cancer. Zhong Liu Fang Zhi Za Zhi. 2005;12(5):383-384.

59. Xie CY, Lou SY, Xu H. The efficacy of capecitabine in treatment with 5-fluorouracil-resistant patients with advanced colorectal cancer. Jiangxi Yixueyuan Xuebao. 2005;45(5):163-164. Chinese.

60. Zhou JF, Bai CM, Cheng YJ, Jia N, Shao YJ, Chen SC. [Efficacy and safety of combination of irinotecan and capecitabine in patients with metastatic colorectal cancer after failure of chemotherapy with oxaliplatin]. Zhongguo Yi Xue Ke Xue Yuan Xue Bao. 2009;31(3): 358-361. Chinese.

61. Li X, Deng LY. Efficacy and safety of irinotecan combined with capecitabine regimen in patients with advanced colorectal cancer after failure of chemotherapy with oxaliplatin. Jiangxi Med Pharmacol. 2007;42(8): 719-721. Chinese. 
62. Xu CN. Weekly irinotecan combined with capecitabine as second-line chemotherapy in the treatment of advanced colorectal cancer. Chin Clin Oncol. 2008;13(7):651-653. Chinese.

63. Wu XN, Zhao YB, Wu JY, et al. Efficacy and safety of bevacizumab plus capecitabine for metastatic colorectal cancer. Zhongguo Yi Xue Ke Xue Yuan Xue Bao. 2010;32(4):417-420.

64. Zhuang CP, Cai GY, Li YH, Wang YQ, Chen WY. Efficacies of capecitabine combined with irinotecan or rh-endostatin for treatment of oxaliplatin-resistant patients with advanced colorectal cancer. Chin J Cancer Biother. 2009;16(2):175-180.

65. Wang SG, Zhang DW. Clinical observation on capecitabine monotherapy for elderly patients with advanced colon cancer. Anhui Med J. 2009;30(10):1166-1167.

66. Zhao JG, Xiong JP, Xiang XJ, Qiu F, Zhang L, Yu F. Capecitabine combined with oxaliplatin as first-line chemotherapy in 58 cases with advanced colorectal cancer in elderly. Chin J Cancer. 2008;14(11): 929-931.

67. Gao L, Wang YX, Lu LQ, Zhao TW. Clinical study of capecitabine combined regimen in treatment of advanced colorectal cancer in senile patients. Chin J Postgrad Med. 2008;31(8):20-23.

68. Bi JW, Wang BH, Liu X, Di JS, Shi J. Oxaliplatin combined with capecitabine in the treatment of metastatic colorectal cancer in senile patients. Shandong Med Pharmocol J. 2009;49(14):81-82. Chinese.
69. Tse VC, Ng WT, Lee V, et al. Cost-analysis of XELOX and FOLFOX4 for treatment of colorectal cancer to assist decisionmaking on reimbursement. BMC Cancer. 2011;11:288.

70. Wu WZ, Wu B. Oxaliplatin combined capecitabine in the treatment of 43 cases of advanced colorectal cancer. Med J Commun. 2012;26(3): 242-245. Chinese.

71. Luo YZ, Ouyang Z. Efficacy and side effect of XELOX and FOLFOX6 as first-line chemotherapy on metastatic colorectaI cancer. Mod Oncol. 2010;18(1):125-127. Chinese.

72. Yue XL, Liu WM. Comparative study of drug combination of CPT-11 plus $\mathrm{CAP}$ and 5-Fu/CF in the treatment of advanced colorectal cancer. Pract Oncol. 2009;23(4):336-362. Chinese.

73. Zhao R, Zhu J, Ji X, et al. A phase II study of irinotecan and capecitabine for patients with unresectable liver-only metastases from colorectal cancer. Jpn J Clin Oncol. 2010;40(1):10-16.

74. Geng M, Ma T, Xu HP, et al. The combination of capecitabine and irinotecan in treating 38 cases of advanced colorectaI cancer as secondIine chemotherapy regimen. Mod Oncol. 2009;17(7):1305-1307. Chinese.
OncoTargets and Therapy

\section{Publish your work in this journal}

OncoTargets and Therapy is an international, peer-reviewed, open access journal focusing on the pathological basis of all cancers, potential targets for therapy and treatment protocols employed to improve the management of cancer patients. The journal also focuses on the impact of management programs and new therapeutic agents and protocols on

\section{Dovepress}

patient perspectives such as quality of life, adherence and satisfaction. The manuscript management system is completely online and includes a very quick and fair peer-review system, which is all easy to use. Visit http://www.dovepress.com/testimonials.php to read real quotes from published authors. 